\title{
Minimum Clique Number, Chromatic Number, and Ramsey Numbers
}

\author{
Gaku Liu \\ Department of Mathematics \\ Princeton University \\ Princeton, NJ 08544, USA \\ xueliu@princeton.edu
}

Submitted: Sep 5, 2011; Accepted: Mar 10, 2012; Published: Mar 19, 2012

Mathematics Subject Classifications: 05C35, 05C69, 05D10

\begin{abstract}
Let $Q(n, c)$ denote the minimum clique number over graphs with $n$ vertices and chromatic number $c$. We investigate the asymptotics of $Q(n, c)$ when $n / c$ is held constant. We show that when $n / c$ is an integer $\alpha, Q(n, c)$ has the same growth order as the inverse function of the Ramsey number $R(\alpha+1, t)$ (as a function of $t$ ). Furthermore, we show that if certain asymptotic properties of the Ramsey numbers hold, then $Q(n, c)$ is in fact asymptotically equivalent to the aforementioned inverse function. We use this fact to deduce that $Q(n,\lceil n / 3\rceil)$ is asymptotically equivalent to the inverse function of $R(4, t)$.
\end{abstract}

\section{Introduction}

In this paper, all graphs are assumed to be simple and finite. Let $\omega(G), \alpha(G)$, and $\chi(G)$ denote the clique number, independence number, and chromatic number, respectively, of a graph $G$. We are concerned with the following problem: Given the chromatic number of a graph, what is the minimum possible clique number? It is well-known that given any $c$, there exist graphs with $\Theta\left(c^{2} \log c\right)$ vertices which have chromatic number $c$ and clique number 2 . On the other hand, when the chromatic number is "large" compared to the number of vertices, larger cliques are forced. In particular, this happens when the chromatic number is a constant multiple of the number of vertices. We will focus on this case.

We define

$$
Q(n, c)=\min \{\omega(G):|V(G)|=n \text { and } \chi(G)=c\} .
$$

The problem of showing the existence of large cliques in graphs with high chromatic number is similar to certain problems studied in Ramsey theory. Classical Ramsey theory 
can be thought of as studying the existence of large cliques in graphs with bounded independence number; i.e., the determination of the function

$$
\omega(n, \alpha)=\min \{\omega(G):|V(G)|=n \text { and } \alpha(G) \leq \alpha\} .
$$

(Note the relationship between this function and the classical Ramsey numbers $R(s, t)$; specifically, $\omega(n, \alpha)$ is the unique integer $\omega$ such that $R(\alpha+1, \omega) \leq n<R(\alpha+1, \omega+1)$.) There is a clear analogy between definitions (1) and (2), and in fact, the two functions appear to be closely related. The relationship was pointed to in [6], where authors Gyárfás, Sebö, and Trotignon studied the chromatic gap defined by $\operatorname{gap}(n)=\max \{\chi(G)-\omega(G)$ : $|V(G)|=n\}$. In the case where $c \geq(n+3) / 2$, an exact relationship between $Q(n, c)$ and $\omega(n, 2)$ was given by Biró, Füredi, and Jahanbekam [2]:

Theorem 1.1 (Biró, Füredi, Jahanbekam). For $n \geq 2 k+3$,

$$
Q(n, n-k)=n-2 k+\min \sum_{i=1}^{s}\left(\omega\left(2 k_{i}+1,2\right)-1\right)
$$

where the minimum is taken over all positive integers $k_{1}, \ldots, k_{s}$ with $k_{1}+\cdots+k_{s}=k$. Furthermore, in the minimum we can take $s \leq 3$.

Theorem 1.1 thus establishes a connection between the numbers $Q(n, c)$ and the Ramsey numbers $R(3, t)$ in the case where (roughly) $c>n / 2$. The authors of [2] posed the problem of finding the relationship between the Ramsey numbers and the numbers $Q(n,\lceil\tau n\rceil)$ for $\tau \leq 1 / 2$.

In this paper, we look at the asymptotic behavior of $Q(n,\lceil n / \alpha\rceil)$ when $\alpha$ is an integer. We show that the behavior of this function is closely related to the behavior of $\omega(n, \alpha)$. Specifically, we have the following.

Theorem 1.2. For each positive integer $\alpha$, there exists a constant $0<c_{\alpha} \leq 1$ such that

$$
c_{\alpha} \omega(n, \alpha) \leq Q(n,\lceil n / \alpha\rceil) \leq \omega(n, \alpha) .
$$

for all $n$.

It seems, however, that one can do better than this. We also prove the following.

Theorem 1.3. Let $\alpha \geq 2$ be an integer, and suppose there exists a function $\kappa: \mathbb{N} \rightarrow \mathbb{N}$ such that

$$
\lim _{n \rightarrow \infty} \frac{\omega(\kappa(n), \alpha-1)}{\omega(n, \alpha)}=\infty \quad \text { and } \quad \lim _{n \rightarrow \infty} \frac{\omega(\kappa(n), \alpha)}{\omega(n, \alpha)}=0 .
$$

Then

$$
\lim _{n \rightarrow \infty} \frac{Q(n,\lceil n / \alpha\rceil)}{\omega(n, \alpha)}=1
$$

As a consequence of Theorem 1.3 and known bounds on the Ramsey numbers $R(3, t)$ and $R(4, t)$, we have the following corollary. 


\section{Corollary 1.4.}

$$
\lim _{n \rightarrow \infty} \frac{Q(n,\lceil n / 3\rceil)}{\omega(n, 3)}=1 .
$$

The problem of determining the asymptotic behavior of $Q(n,\lceil n / \alpha\rceil)$ is thus partly reduced to determining the asymptotic behavior of $\omega(n, \alpha)$ (i.e., the Ramsey numbers).

\section{Ramsey Numbers and Asymptotics}

Before proving these theorems, we introduce some necessary background on Ramsey numbers. The Ramsey number $R(s, t)$ is the minimum integer $R$ such that every graph with at least $R$ vertices has either an independent set of size $s$ or a clique of size $t$. There have been a number of results on the asymptotics of $R(s, t)$ when $s$ is fixed and $t$ varies. Atjai, Komlós, and Szemerédi [1] proved that for every fixed $s \geq 3$,

$$
R(s, t)=O\left(\frac{t^{s-1}}{(\log t)^{s-2}}\right) .
$$

For $s=3$, the lower bound

$$
R(3, t)=\Omega\left(\frac{t^{2}}{\log t}\right)
$$

was first proved by Kim [7]. More generally, Bohman and Keevash [3], [4] proved that for every fixed $s \geq 3$,

$$
R(s, t)=\Omega\left(\frac{t^{\frac{s+1}{2}}}{(\log t)^{\frac{s+1}{2}-\frac{1}{s-2}}}\right) .
$$

Our results will make use of an inverse Ramsey function, which we mentioned earlier and whose definition we repeat: For each positive integer $\alpha$, let

$$
\omega(n, \alpha)=\min \{\omega(G):|V(G)|=n \text { and } \alpha(G) \leq \alpha\} .
$$

In other words, $\omega(n, \alpha)$ is the unique integer $\omega$ such that $R(\alpha+1, \omega) \leq n<R(\alpha+1, \omega+1)$. For fixed $\alpha$, we can therefore view $\omega(n, \alpha)$ as the inverse function for $R(\alpha+1, t)$. The bounds (3) and (4) imply that for each $\alpha \geq 2$, there exist constants $k_{\alpha}, k_{\alpha}^{\prime}$ such that

$$
k_{\alpha} n^{1 / \alpha}(\log n)^{\beta} \leq \omega(n, \alpha) \leq k_{\alpha}^{\prime} n^{2 /(\alpha+2)}(\log n)^{\beta^{\prime}} .
$$

where $\beta=(\alpha-1) / \alpha$ and $\beta^{\prime}=1-2 /[(\alpha-1)(\alpha+2)]$. In particular, we have

$$
k_{2} n^{1 / 2}(\log n)^{1 / 2} \leq \omega(n, 2) \leq k_{2}^{\prime} n^{1 / 2}(\log n)^{1 / 2}
$$

and

$$
k_{3} n^{1 / 3}(\log n)^{2 / 3} \leq \omega(n, 3) \leq k_{3}^{\prime} n^{2 / 5}(\log n)^{4 / 5} .
$$

Finally, we note that for all $m, n$, and $\alpha$,

$$
\omega(m, \alpha)+\omega(n, \alpha) \geq \omega(m+n, \alpha) .
$$


This follows because if $G_{1}, G_{2}$ are graphs with $\left|V\left(G_{1}\right)\right|=m,\left|V\left(G_{2}\right)\right|=n, \alpha\left(G_{1}\right), \alpha\left(G_{2}\right) \leq$ $\alpha$, and $\omega\left(G_{1}\right)=\omega(m, \alpha), \omega\left(G_{2}\right)=\omega(n, \alpha)$, then the join $G=G_{1}+G_{2}$ of the two graphs (i.e., the graph formed by taking the disjoint union of $G_{1}$ and $G_{2}$ and adding every edge between a vertex of $G_{1}$ and a vertex of $\left.G_{2}\right)$ is a graph with $V(G)=m+n, \alpha(G) \leq \alpha$, and $\omega(G)=\omega(m, \alpha)+\omega(n, \alpha)$.

\section{Proof of Theorem 1.2}

We now proceed with the proofs of our main results. We restate our first result.

Theorem 1.2. For each positive integer $\alpha$, there exists a constant $0<c_{\alpha} \leq 1$ such that

$$
c_{\alpha} \omega(n, \alpha) \leq Q(n,\lceil n / \alpha\rceil) \leq \omega(n, \alpha) .
$$

for all $n$.

Proof. We first establish the upper bound. Fix $\alpha$. Let $G$ be a graph with $|V(G)|=n$, $\alpha(G) \leq \alpha$, and $\omega(G)=\omega(n, \alpha)$. From the inequality $\chi(G) \geq|V(G)| / \alpha(G)$, we have $\chi(G) \geq\lceil n / \alpha\rceil$. Delete edges one by one from $G$ until we have a graph $G^{\prime}$ with $\chi\left(G^{\prime}\right)=$ $\lceil n / \alpha\rceil$. The clique number does not increase as we delete edges, so $\omega\left(G^{\prime}\right) \leq \omega(n, \alpha)$. Since $G^{\prime}$ has $n$ vertices, this establishes $Q(n,\lceil n / \alpha\rceil) \leq \omega(n, \alpha)$.

To prove the lower bound, we induct on $\alpha$. For $\alpha=1$, we have $Q(n, n)=\omega(n, 1)=n$, and the proof is done with $c_{1}=1$. Now, fix $\alpha \geq 2$, and suppose there exists $0<c_{\alpha-1} \leq 1$ such that

$$
c_{\alpha-1} \omega(n, \alpha-1) \leq Q(n,\lceil n /(\alpha-1)\rceil)
$$

for all $n$. We will prove that for sufficiently large $n$,

$$
\frac{1}{5} c_{\alpha-1} \omega(n, \alpha) \leq Q(n,\lceil n / \alpha\rceil)
$$

which will complete the induction.

Suppose $G_{0}$ is a graph with $\left|V\left(G_{0}\right)\right|=n$ and $\chi\left(G_{0}\right)=\lceil n / \alpha\rceil$. It suffices to show that for any such $G_{0}$ with sufficiently large $n$, we have $\omega\left(G_{0}\right) \geq \frac{1}{5} c_{\alpha-1} \omega(n, \alpha)$. If $G_{0}$ contains an independent set of size $\alpha+1$, delete it to obtain a graph $G_{1}$. If $G_{1}$ has an independent set of size $\alpha+1$, delete it to obtain $G_{2}$. Continue doing this until we have a graph $G_{t}$ $(t \geq 0)$ such that either

(a) $\alpha\left(G_{t}\right) \leq \alpha$, or

(b) $t>n /(2 \alpha)$.

The clique number does not increase as we delete vertices, so $\omega\left(G_{0}\right) \geq \omega\left(G_{t}\right)$. It thus suffices to show that $\omega\left(G_{t}\right) \geq \frac{1}{5} c_{\alpha-1} \omega(n, \alpha)$. Also, the chromatic number decreases by at most one each time we delete an independent set, so $\chi\left(G_{t}\right) \geq \chi(G)-t=\lceil n / \alpha\rceil-t$. 
First, suppose that $t \leq n /(2 \alpha)$. Then we must have case (a), so by the definition of $\omega(\cdot, \cdot)$,

$$
\omega\left(G_{t}\right) \geq \omega\left(\left|V\left(G_{t}\right)\right|, \alpha\right) .
$$

Now,

$$
\left|V\left(G_{t}\right)\right|=n-(\alpha+1) t \geq n-\frac{(\alpha+1) n}{2 \alpha}=\left(\frac{1}{2}-\frac{1}{2 \alpha}\right) n .
$$

Since $\alpha \geq 2$, we thus have

$$
\left|V\left(G_{t}\right)\right| \geq \frac{1}{4} n
$$

Combined with (8), and since $\omega(n, \alpha)$ is a non-decreasing function of $n$ (given a graph $G$ with $n+1$ vertices, $\alpha(G) \leq \alpha$, and $\omega(G)=\omega(n+1, \alpha)$, we can delete a vertex to create a graph $G^{\prime}$ with $n$ vertices, $\alpha\left(G^{\prime}\right) \leq \alpha$, and $\omega\left(G^{\prime}\right) \leq \omega(n+1, \alpha)$ ), we have

$$
\omega\left(G_{t}\right) \geq \omega(\lceil n / 4\rceil, \alpha) .
$$

Using property $(7)$ and the monotonicity of $\omega(\cdot, \alpha)$, we thus have

$$
\omega\left(G_{t}\right) \geq \frac{1}{4} \omega(n, \alpha) \geq \frac{1}{5} c_{\alpha-1} \omega(n, \alpha)
$$

as desired.

Now suppose that $t>n /(2 \alpha)$. Notice that

$$
\frac{\lceil n / \alpha\rceil-x}{n-(\alpha+1) x}
$$

is an increasing function of $x$ for $x<n /(\alpha+1)$. Indeed, for $x, y<n /(\alpha+1)$, we have

$$
\begin{aligned}
\frac{\lceil n / \alpha\rceil-x}{n-(\alpha+1) x} & >\frac{\lceil n / \alpha\rceil-y}{n-(\alpha+1) y} \\
\Leftrightarrow(\lceil n / \alpha\rceil-x)(n-(\alpha+1) y) & >(\lceil n / \alpha\rceil-y)(n-(\alpha+1) x) \\
\Leftrightarrow((\alpha+1)\lceil n / \alpha\rceil-n) x & >((\alpha+1)\lceil n / \alpha\rceil-n) y \\
\Leftrightarrow x & >y
\end{aligned}
$$

since $(\alpha+1)\lceil n / \alpha\rceil-n>0$. Thus, we have

$$
\begin{aligned}
\frac{\chi\left(G_{t}\right)}{\left|V\left(G_{t}\right)\right|} & \geq \frac{\lceil n / \alpha\rceil-t}{n-(\alpha+1) t} \\
& >\frac{\lceil n / \alpha\rceil-n /(2 \alpha)}{n-(\alpha+1)[n /(2 \alpha)]} \\
& \geq \frac{n / \alpha-n /(2 \alpha)}{n-(\alpha+1)[n /(2 \alpha)]} \\
& =\frac{1}{\alpha-1} .
\end{aligned}
$$


It follows that

$$
\chi\left(G_{t}\right) \geq\left\lceil\frac{\left|V\left(G_{t}\right)\right|}{\alpha-1}\right\rceil .
$$

Delete edges from $G_{t}$ until we have a graph $G_{t}^{\prime}$ with

$$
\chi\left(G_{t}^{\prime}\right)=\left\lceil\frac{\left|V\left(G_{t}\right)\right|}{\alpha-1}\right\rceil .
$$

By the inductive hypothesis, we thus have

$$
\omega\left(G_{t}\right) \geq \omega\left(G_{t}^{\prime}\right) \geq c_{\alpha-1} \omega\left(\left|V\left(G_{t}\right)\right|, \alpha-1\right) .
$$

Since $\omega\left(\left|V\left(G_{t}\right)\right|, \alpha-1\right) \geq \omega\left(\left|V\left(G_{t}\right)\right|, \alpha\right)$ by the definition of $\omega(\cdot, \cdot)$, it follows that

$$
\omega\left(G_{t}\right) \geq c_{\alpha-1} \omega\left(\left|V\left(G_{t}\right)\right|, \alpha\right) .
$$

Now, by construction, $t$ is the first integer greater than $n /(2 \alpha)$, so $t \leq n /(2 \alpha)+1$. Thus,

$$
\left|V\left(G_{t}\right)\right|=n-(\alpha+1) t \geq n-\frac{(\alpha+1) n}{2 \alpha}-(\alpha+1)=\left(\frac{1}{2}-\frac{1}{2 \alpha}\right) n-(\alpha+1) .
$$

Since $\alpha \geq 2$, we have $\left|V\left(G_{t}\right)\right| \geq \frac{1}{4} n-(\alpha+1)$. Hence, for sufficiently large $n$, we have $\left|V\left(G_{t}\right)\right| \geq \frac{1}{5} n$. From (9) and property (7), we thus have

$$
\omega\left(G_{t}\right) \geq c_{\alpha-1} \omega(\lceil n / 5\rceil, \alpha) \geq \frac{1}{5} c_{\alpha-1} \omega(n, \alpha)
$$

which completes the proof.

\section{Proof of Theorem 1.3}

Theorem 1.3. Let $\alpha \geq 2$ be an integer, and suppose there exists a function $\kappa: \mathbb{N} \rightarrow \mathbb{N}$ such that

$$
\lim _{n \rightarrow \infty} \frac{\omega(\kappa(n), \alpha-1)}{\omega(n, \alpha)}=\infty \quad \text { and } \quad \lim _{n \rightarrow \infty} \frac{\omega(\kappa(n), \alpha)}{\omega(n, \alpha)}=0 .
$$

Then

$$
\lim _{n \rightarrow \infty} \frac{Q(n,\lceil n / \alpha\rceil)}{\omega(n, \alpha)}=1
$$

Proof. Fix $\alpha \geq 2$, and suppose $\kappa$ is a function as described. We have $\lim _{n \rightarrow \infty} \kappa(n)=\infty$ because $\lim _{n \rightarrow \infty} \omega(\kappa(n), \alpha-1) / \omega(n, \alpha)=\infty$. Also, we have $\lim _{n \rightarrow \infty} \kappa(n) / n=0$, since $\lim _{n \rightarrow \infty} \omega(\kappa(n), \alpha) / \omega(n, \alpha)=0$ and

$$
\frac{\omega(\kappa(n), \alpha)}{\omega(n, \alpha)} \geq \frac{\omega(\kappa(n), \alpha)}{\lceil n / \kappa(n)\rceil \omega(\kappa(n), \alpha)}=\frac{1}{\lceil n / \kappa(n)\rceil} \geq 2(\kappa(n) / n)
$$

where the first inequality holds by property (7). 
We will prove that for sufficiently large $n$,

$$
\omega(n-(\alpha+1) \kappa(n), \alpha) \leq Q(n,\lceil n / \alpha\rceil) \leq \omega(n, \alpha) .
$$

Note that we may assume $n-(\alpha+1) \kappa(n)>0$ because $\lim _{n \rightarrow \infty} \kappa(n) / n=0$. Proving (10) will suffice to prove Theorem 1.3 , because by property $(7)$ we have

$$
\omega(n-(\alpha+1) \kappa(n), \alpha) \geq \omega(n, \alpha)-(\alpha+1) \omega(\kappa(n), \alpha)
$$

and hence

$$
\frac{\omega(n-(\alpha+1) \kappa(n), \alpha)}{\omega(n, \alpha)} \geq 1-(\alpha+1) \frac{\omega(\kappa(n), \alpha)}{\omega(n, \alpha)} \rightarrow 1 \quad \text { as } n \rightarrow \infty
$$

by the definition of $\kappa$.

The upper bound of (10) was established in the previous section. For the lower bound, let $n$ be large enough so that $n-(\alpha+1) \kappa(n)>0$, and suppose $G_{0}$ is a graph with $\left|V\left(G_{0}\right)\right|=n$ and $\chi\left(G_{0}\right)=\lceil n / \alpha\rceil$. It suffices to show that for any such $G_{0}$ with sufficiently large $n$, we have $\omega\left(G_{0}\right) \geq \omega(n-(\alpha+1) \kappa(n), \alpha)$. If $G_{0}$ contains an independent set of size $\alpha+1$, delete it to obtain a graph $G_{1}$. If $G_{1}$ contains an independent set of size $\alpha+1$, delete it to obtain $G_{2}$. Continue doing this until we have a graph $G_{t}$ such that either

(a) $\alpha\left(G_{t}\right) \leq \alpha$, or

(b) $t=\kappa(n)$.

Since $\omega(G) \geq \omega\left(G_{t}\right)$, it suffices to show $\omega\left(G_{t}\right) \geq \omega(n-(\alpha+1) \kappa(n), \alpha)$. Because the chromatic number decreases by at most one each time we remove an independent set, we have $\chi\left(G_{t}\right) \geq\lceil n / \alpha\rceil-t$.

If $t<\kappa(n)$, then we must have case (a). Thus, we have

$$
\omega\left(G_{t}\right) \geq \omega\left(\left|V\left(G_{t}\right)\right|, \alpha\right)=\omega(n-(\alpha+1) t, \alpha) \geq \omega(n-(\alpha+1) \kappa(n), \alpha)
$$

as desired.

Now suppose that $t=\kappa(n)$. We have $\left|V\left(G_{t}\right)\right|=n-(\alpha+1) \kappa(n)$ and $\chi\left(G_{t}\right) \geq$ $\lceil n / \alpha\rceil-\kappa(n)$. Let $H_{0}=G_{t}$. If $\chi\left(H_{0}\right) /\left|V\left(H_{0}\right)\right|<1 /(\alpha-1)$, then by the inequality $\alpha\left(H_{0}\right) \geq\left|V\left(H_{0}\right)\right| / \chi\left(H_{0}\right)$, we have $\alpha\left(H_{0}\right) \geq \alpha$. Hence, if $\chi\left(H_{0}\right) /\left|V\left(H_{0}\right)\right|<1 /(\alpha-1)$, we can remove an independent set of size $\alpha$ from $H_{0}$ to obtain a graph $H_{1}$. If $\chi\left(H_{1}\right) /\left|V\left(H_{1}\right)\right|<$ $1 /(\alpha-1)$, we again remove an independent set of size $\alpha$ to obtain a graph $H_{2}$. Continue this process until we have a graph $H_{s}$ with

$$
\frac{\chi\left(H_{s}\right)}{\left|V\left(H_{s}\right)\right|} \geq \frac{1}{\alpha-1}
$$

To see that we will indeed reach such an $s$, note that at each $H_{i}$ we have $\chi\left(H_{i}\right) /\left|V\left(H_{i}\right)\right| \geq$ $f(i)$, where

$$
f(x)=\frac{\lceil n / \alpha\rceil-\kappa(n)-x}{n-(\alpha+1) \kappa(n)-\alpha x} .
$$


Then $f$ is an increasing function of $x$ for $x<n / \alpha-\frac{\alpha+1}{\alpha} \kappa(n)$; indeed, for $x, y$ in this range, we have

$$
\begin{aligned}
\frac{\lceil n / \alpha\rceil-\kappa(n)-x}{n-(\alpha+1) \kappa(n)-\alpha x} & <\frac{\lceil n / \alpha\rceil-\kappa(n)-y}{n-(\alpha+1) \kappa(n)-\alpha y} \\
\Leftrightarrow(\alpha\lceil n / \alpha\rceil-\alpha \kappa(n)-n+(\alpha+1) \kappa(n)) x & <(\alpha\lceil n / \alpha\rceil-\alpha \kappa(n)-n+(\alpha+1) \kappa(n)) y \\
\Leftrightarrow x & <y
\end{aligned}
$$

since

$$
\alpha\lceil n / \alpha\rceil-\alpha \kappa(n)-n+(\alpha+1) \kappa(n)=\alpha\lceil n / \alpha\rceil-n+\kappa(n) \geq \kappa(n)>0 .
$$

In addition, if $\lceil n / \alpha-2 \kappa(n)\rceil<n / \alpha-\frac{\alpha+1}{\alpha} \kappa(n)$, then

$$
\begin{aligned}
f(\lceil n / \alpha-2 \kappa(n)\rceil) & \geq f(n / \alpha-2 \kappa(n)) \\
& =\frac{\lceil n / \alpha\rceil-\kappa(n)-(n / \alpha-2 \kappa(n))}{n-(\alpha+1) \kappa(n)-\alpha(n / \alpha-2 \kappa(n))} \\
& \geq \frac{n / \alpha-\kappa(n)-(n / \alpha-2 \kappa(n))}{n-(\alpha+1) \kappa(n)-\alpha(n / \alpha-2 \kappa(n))} \\
& =\frac{1}{\alpha-1} .
\end{aligned}
$$

Thus, as long as $\lceil n / \alpha-2 \kappa(n)\rceil<n / \alpha-\frac{\alpha+1}{\alpha} \kappa(n)$, the sequence $H_{0}, H_{1}, H_{2}, \ldots$ will stop at an $H_{s}$ with $s \leq\lceil n / \alpha-2 \kappa(n)\rceil$. Since $\alpha \geq 2$ and $\kappa(n) \geq 2$ for large enough $n$, we have $n / \alpha-2 \kappa(n) \leq n / \alpha-\frac{\alpha+1}{\alpha} \kappa(n)-1$, and hence $\lceil n / \alpha-2 \kappa(n)\rceil<n / \alpha-\frac{\alpha+1}{\alpha} \kappa(n)$, as desired.

We thus have a graph $H_{s}$ with $\omega\left(G_{t}\right) \geq \omega\left(H_{s}\right), s \leq\lceil n / \alpha-2 \kappa(n)\rceil$, and

$$
\chi\left(H_{s}\right) \geq\left\lceil\frac{\left|V\left(H_{s}\right)\right|}{\alpha-1}\right\rceil .
$$

Delete edges from $H_{s}$ until we have a graph $H_{s}^{\prime}$ with

$$
\chi\left(H_{s}^{\prime}\right)=\left\lceil\frac{\left|V\left(H_{s}\right)\right|}{\alpha-1}\right\rceil .
$$

By Theorem 1.2, we thus have

$$
\omega\left(G_{t}\right) \geq \omega\left(H_{s}^{\prime}\right) \geq c_{\alpha-1} \omega\left(\left|V\left(H_{s}\right)\right|, \alpha-1\right) .
$$

for some $c_{\alpha-1}>0$. Now, since $s \leq\lceil n / \alpha-2 \kappa(n)\rceil$, we have

$$
\begin{aligned}
\left|V\left(H_{s}\right)\right| & =n-(\alpha+1) \kappa(n)-\alpha s \\
& \geq n-(\alpha+1) \kappa(n)-\alpha\lceil n / \alpha-2 \kappa(n)\rceil \\
& \geq n-(\alpha+1) \kappa(n)-\alpha(n / \alpha-2 \kappa(n)+1) \\
& =(\alpha-1) \kappa(n)-\alpha
\end{aligned}
$$


and hence

$$
\omega\left(G_{t}\right) \geq c_{\alpha-1} \omega((\alpha-1) \kappa(n)-\alpha, \alpha-1) .
$$

Applying $\alpha \geq 2$ and then property (7), we have

$$
\begin{aligned}
\omega\left(G_{t}\right) & \geq c_{\alpha-1} \omega(\kappa(n)-\alpha, \alpha-1) \\
& \geq c_{\alpha-1}(\omega(\kappa(n), \alpha-1)-\omega(\alpha, \alpha-1)) \\
& =c_{\alpha-1}(\omega(\kappa(n), \alpha-1)-2) \\
& \geq \frac{1}{2} c_{\alpha-1} \omega(\kappa(n), \alpha-1)
\end{aligned}
$$

for large enough $n$. Now, since

$$
\lim _{n \rightarrow \infty} \frac{\omega(\kappa(n), \alpha-1)}{\omega(n, \alpha)}=\infty
$$

we have for large enough $n$

$$
\omega\left(G_{t}\right) \geq \frac{1}{2} c_{\alpha-1} \omega(\kappa(n), \alpha-1) \geq \omega(n, \alpha) \geq \omega(n-(\alpha+1) \kappa(n), \alpha)
$$

which completes the proof.

Corollary 1.4 .

$$
\lim _{n \rightarrow \infty} \frac{Q(n,\lceil n / 3\rceil)}{\omega(n, 3)}=1 .
$$

Proof. Let $\kappa(n)=\left\lceil n^{49 / 60}\right\rceil$. From (5) and (6), we have

$$
\frac{\omega\left(\left\lceil n^{49 / 60}\right\rceil, 2\right)}{\omega(n, 3)} \geq \frac{k_{2}\left(n^{49 / 60}\right)^{1 / 2}\left(\log n^{49 / 60}\right)^{1 / 2}}{k_{3}^{\prime} n^{2 / 5}(\log n)^{4 / 5}} \rightarrow \infty \text { as } n \rightarrow \infty
$$

since $(49 / 60)(1 / 2)>(48 / 60)(1 / 2)=2 / 5$. Also,

$$
\frac{\omega\left(\left[n^{49 / 60}\right\rceil, 3\right)}{\omega(n, 3)} \leq \frac{k_{3}^{\prime}\left(n^{49 / 60}+1\right)^{2 / 5}\left[\log \left(n^{49 / 60}+1\right)\right]^{4 / 5}}{k_{3} n^{1 / 3}(\log n)^{2 / 3}} \rightarrow 0 \text { as } n \rightarrow \infty
$$

because $(49 / 60)(2 / 5)<(50 / 60)(2 / 5)=1 / 3$. Thus, the desired result holds by Theorem 1.3 .

It may be the case that there exists an appropriate $\kappa(n)$ for each $\alpha \geq 2$. For example, it is conjectured that for any fixed $s \geq 3$, we have

$$
R(s, t)=t^{s-1+o(1)} .
$$

If this is the case, then for a given $\alpha \geq 2$ we can take $\kappa(n)=\left\lceil n^{\gamma}\right\rceil$, where $\frac{\alpha-1}{\alpha}<\gamma<1$. We would then have $\lim _{n \rightarrow \infty} Q(n,\lceil n / \alpha\rceil) / \omega(n, \alpha)=1$ for any $\alpha \geq 2$. 


\section{$5 \quad$ Further Questions}

We have proven for positive integer $\alpha$ that $Q(n,\lceil n / \alpha\rceil)$ and $\omega(n, \alpha)$ have the same order of growth, and are quite possibly asymptotically equivalent. In the case $\alpha=2$, however, one can use the techniques of [2] to show

$$
\omega(n, 2)-2 \leq Q(n,\lceil n / 2\rceil) \leq \omega(n, 2) .
$$

Furthermore, one can prove that for almost all values of $n$, we in fact have $Q(n,\lceil n / 2\rceil)=$ $\omega(n, 2)$. (See [6] for a more precise statement.) Is there a similar, more exact relationship between $Q(n,\lceil n / \alpha\rceil)$ and $\omega(n, \alpha)$ for $\alpha \geq 3$ ? More precisely, one can ask the following questions.

Question 1. For each $\alpha$, is $\omega(n, \alpha)-Q(n,\lceil n / \alpha\rceil)$ bounded?

Question 2. Do there exist infinitely many $n$ for which $Q(n,\lceil n / \alpha\rceil)=\omega(n, \alpha)$ ?

Finally, it would be nice to have a better understanding of the behavior of $Q(n,\lceil n / \alpha\rceil)$ when $\alpha$ is not an integer.

\section{Acknowledgments}

This research was conducted at the summer REU at the University of Minnesota Duluth supervised by Joe Gallian, funded by NSF/DMS grant 1062709 and NSA grant H9823011-1-0224. The author would like to thank Joe Gallian, the advisers Jonathan Wang and Alan Deckelbaum, and the participants of the program.

\section{References}

[1] Miklós Atjai, János Komlós, and Endre Szemerédi, A note on Ramsey numbers, J. Combin. Theory Ser. A 29 (1980), no. 3, 354-360.

[2] Csaba Biró, Zoltán Füredi, and Sogol Jahanbekam, Large Chromatic Number and Ramsey Graphs, (2011), arXiv:1103.3917v2.

[3] Tom Bohman, The triangle-free process, Adv. Math. 221 (2009), 1653-1677.

[4] Tom Bohman, Peter Keevash, The early evolution of the $H$-free process, Invent. Math. 181 (2010), 291-336.

[5] Ronald Graham, Bruce Rothschild, and Joel Spencer, Ramsey Theory, John Wiley \& Sons, 2 ed., 1990.

[6] András Gyárfás, András Sebő, Nicolas Trotignon, The chromatic gap and its extremes, (2011), arXiv:1108.3444v1.

[7] Jeong Han Kim, The Ramsey Number $R(3, t)$ has Order of Magnitude $t^{2} / \log t$, Random Structures Algorithms 7 (1995), no. 3, 173-207. 